\title{
PRESSÃO ARTERIAL CENTRAL E RISCO CARDIOVASCULAR
}

\author{
CENTRAL BLOOD PRESSURE AND CARDIOVASCULAR RISK
}

Lucélia Batista Cunha Magalhães ${ }^{1}$, Magno Conceição das Mercês ${ }^{1,2}$, Rodrigo Fernandes Weyll Pimentel ${ }^{3}$

\section{RESUMO}

Pressão Central, como o nome indica, é uma medida hemodinâmica semelhante a pressão arterial convencional porem avaliada de forma indireta por equipamento especifico, que avalia estes parâmetros na saída do sangue na raiz da aorta. Esta medida tem uma maior confiabilidade pois prediz de forma mais acurada os riscos de adoecimento e morte cardiovascular. Isto ocorre, pois a a onda de pulso (OP) ao percorrer os trajetos arteriais sofrem ampliações e importantes modificações no seu contorno deformando o valor original. Embora seja mais precisa em valores, ainda não é usado de rotina na pratica clinica por razoes de custos dos seus equipamentos e provavelmente por exigir habilidades maiores que as medidas captadas pelo equipamentos de mensuração periférica.

Descritores: Pressão central; Risco Cardiovascular; Fisiologia da Onda de Pulso.

\section{ABSTRACT}

Central pressure, as the name implies, is a hemodynamic measure similar to conventional blood pressure, but indirectly assessed by specific equipment, which evaluates these parameters at the blood outlet at the root of the aorta. This measure has greater confidence because it more accurately predicts the risks of cardiovascular disease and death. This occurs because the pulse wave $(O P)$ when traversing the arterial paths provides enlargements and modifications in its contour, deforming the original value. Although it is more precise in terms of values, it is not yet routinely used in clinical practice for reasons of the cost of its equipment and probably because it requires greater needs than measures captured by peripheral measurement equipment.

Keywords: Central Pressure; Cardiovascular Risk; Pulso Wave Psysiology.

\section{INTRODUÇÃO}

A pressão arterial é uma medida da força que o coração utiliza para bombear o sangue pelo corpo, juntamente com os diferentes tipos de artérias, com o objetivo de manter irrigados todos os órgãos e tecidos. É um componente fundamental da vida. É um mecanismo muito complexo manter o fluxo sanguíneo adequado para todo o organismo, sem desperdício de energia, em momentos muito diferentes e com necessidades que variam desde um sono profundo a uma grande reação de luta ou fuga.

Os componentes mais utilizados habitualmente para expressar essa medida são a pressão arterial sistólica (PAS), a pressão arterial diastólica (PAD), pressão de perfusão tecidual ou pressão média (PM) e a pressão de pulso (PP), seu componente pulsátil propriamente dito. Todos estes componentes descritos têm seus próprios significados e elementos constitutivos e participam de forma peculiar e integrada da onda do pulso (OP). Esta onda possibilita a medição destes componentes entre outros, através de fórmulas. A cada dia tem sido crescente o entendimento sobre a forma do pulso e a sequência destas formas no tempo e os diferentes prognósticos das medidas centrais quando comparadas com as medidas periféricas mais habituais.

Podemos teoricamente medir esta força/pressão em qualquer território de forma invasiva e não invasiva. Na prática clínica, a medimos com equipamentos auscultatórios e oscilométricos não invasivos, na região dos membros superiores e detectamos os valores de PAS e PAD, para então calcularmos a PM e a PP. Outra forma de medição é a visualização da curva com sua impressão e muitos cálculos automaticamente realizados ou não. Estes conhecimentos permitem um melhor entendimento da estrutura e função, além do fluxo como das artérias. ${ }^{1}$

A Hipertensão Arterial Sistêmica (HA), definida como elevação sustentada da pressão (medida habitualmente no braço direito), é um importante fator de risco para doenças cardiovasculares. Sua redução diminui estes eventos de forma consistente. Atualmente, evidências disponíveis sugerem uma maior importância para a redução da PAS do que da PAD. Além disso, a PP (que nada mais é do que a PAS - PAD)

1. Faculdade de Medicina da UnesulBahia- UniFTC, Eunapolis, BA, Brasil.

2. Universidade de Estado da Bahia- UNEB, Salvador, BA. Universidade Salvador- UNIFACS, Salvador, BA, Brasil.

3. UNIFACS, Nova Iguaçu, RJ, Brasil..

Correspondência: Lucélia Batista Cunha Magalhães. luceliamagalhaes@terra.com.br

http://dx.doi.org/10.47870/1519-7522/2021280135-8 
corresponde ao componente pulsátil das grandes arteriais. Ela possibilita, de forma mais acurada, melhores resultados de predição de eventos cardiovasculares e está mais fortemente relacionada ao desgaste do sistema cardiovascular do que a PAS isolada a depender da idade da população estudada. ${ }^{2}$ Existem também evidências de maior importância prognóstica da pressão aórtica central do que braquial quando medidas em pacientes hipertensos tratados ou não. ${ }^{1}$

A PM é relativamente constante em diferentes artérias, porém o mesmo não ocorre com a PAS tanto nas medições centrais quanto nas periféricas. A pressão aórtica central e sistólica braquial e as pressões de pulso podem diferir consideravelmente com base na velocidade da onda de pulso (VOP), quanto do índice de aumentação (Alx), indicadores de rigidez arterial.

As OP, ao percorrer as árvores arteriais, da aorta para a regiões distais, sofrem amplificações e modificações no seu contorno e isso modifica suas medições. Assim, a onda resultante na artéria braquial comparada às pressões avaliadas pela forma do pulso central é diferente. Pressões aórticas centrais devem refletir com mais precisão as condições do trabalho do miocárdico ventricular esquerdo, das artérias coronárias e da vasculatura cerebral e, portanto, em teoria, sendo mais precisas com relação aos danos ocasionados a estes órgãos-alvo e, consequentemente, aos eventos cardiovasculares do que pressões braquiais.

\section{HISTÓRIA}

A interpretação do pulso arterial tem sido uma parte importante do exame médico desde os tempos antigos. O primeiro relato do pulso e sua importância na doença cardiovascular foi documentada há cerca de 4.500 anos. Foram produzidos por médicos chineses ligados ao Imperador Amarelo na publicação clássica de Medicina Interna de Nei Ching. ${ }^{2}$ Nesta frase: "Hence if too much salt is used for food, the pulse hardens" (em tradução livre: "Portanto, se muito sal é usado para o alimento, o pulso endurece"), o pulso "duro", certamente, sugere $\mathrm{HA}$.

O pulso também era algo familiar para chineses, indianos, gregos e médicos romanos que o exploravam regularmente no diagnóstico de doenças. Galeno, um médico grego que viveu no século II a.C., escreveu um livro intitulado "Sobre o prognóstico do pulso", no qual ele descreveu 27 variedades de pulsos. Mais recentemente, após um melhor entendimento do sistema cardiovascular e um estudo sistemático do pulso iniciado no século XVI por Józef Strus', o estudioso e humanista polonês, publicou o livro "Sphygmicae artisiam mille ducentos annos perditae et desideratae Libri V". Em sua obra-prima publicada na Suíça em 1555, Józef Strus' discutiu os tipos de pulso e seu significado clínico. O grande número de tipos de pulso descritos em seu livro reflete o melhor poder de observação dos médicos da época, hoje substituído por instrumentos. Uma abordagem racional para todos os problemas médicos que ele tratou permitiu que ele registrasse um pulso usando curvas análogas às gravadas hoje usando um esfigmógrafo e outro equipamentos através do mesmo princípio.

Métodos gráficos verdadeiros para registro de onda de pulso foram introduzidos muito mais tarde, no século XIX, por Marey, em Paris, e por Mahomed, em Londres. ${ }^{1}$ As formas das ondas de pulso e a velocidade que eles apresentaram parecem surpreendentemente semelhantes aos métodos empregados pela tecnologia mais moderna. São os estudos de Strus' e muitos outros estudiosos notáveis que seguiram seus passos que o tornaram possível iniciar a era moderna da mecânica arterial, suas funções e estruturas.

\section{FISIOLOGIA DA PRESSÃO PERIFÉRICA E DA PRESSÃO CENTRAL}

O sistema arterial é perfeitamente projetado para sua função: recepcionar o sangue em jorros do ventrículo esquerdo a cada batimento cardíaco e impulsioná-lo para frente. Cabe a aorta e as outras grandes artérias de condução recebê-lo e passar adiante para chegar na perfusão tecidual final. Isto ocorre sempre com um fluxo constante e delicado, até as pequenas artérias e arteríolas (vasos de resistência) para todos os tecidos e órgãos do corpo.

A pressão arterial pode ser medida, como já relatado, mais comumente na periferia, em geral no braço esquerdo, ou centralmente através da artéria radial. ${ }^{3}$ Esta medida central não sofre as interferências direta das bifurcações arteriais, das posições posturais maiores, das ateroscleroses periféricas localizadas e da amplificação da onda de pulso ao ser propagada. Assim, parece ser mais estável e, consequentemente, mais fidedigna para avaliar os riscos cardiovasculares.

A pressão arterial (PA) braquial medida com esfigmomanômetro não pode ser considerada equivalente à pressão aórtica, pois esta artéria apresenta valores invariavelmente menores em amplitude, porém com valores pressóricos médios maiores, pois, se assim não fosse, não teria fluxo incidente e progressivo. A PA varia continuamente durante o ciclo cardíaco, embora, na prática, apenas o valor máximo durante a sístole e o valor mínimo durante a diástole sejam medidos, existindo, contudo, muitos outros componentes. Além disso, a forma da onda de pulso varia ao longo da árvore arterial. Com o avanço da onda de pulso das artérias centrais - mais elásticas - para as artérias periféricas - mais rígidas com músculo liso - o pico sistólico se torna mais estreito e mais elevado. Considerando que a PAD e a PM são relativamente constantes, a PAS braquial pode ser $30 \mathrm{mmHg}$ maior do que a pressão sistólica central da aorta em jovens. Esse fenômeno, conhecido como amplificação da pressão sistólica (ou pressão de pulso), ocorre por diversos motivos, entre eles o menor calibre e a maior rigidez das artérias periféricas.

A pressão de pulso varia em toda a árvore arterial, resultando em um gradiente entre a pressão central e periférica. Fatores como idade, frequência cardíaca e altura influenciam esse gradiente. Além disso, os reflexos da onda de pulso ocorrem em vários locais da rede arterial, como áreas com maior gradiente de colágeno / elastina, com maior tônus vasomotor e, principalmente, nos pontos de bifurcação. ${ }^{4}$

\section{PRESSÃO CENTRAL E RISCO CARDIOVASCULAR}

A medida não invasiva da pressão arterial aferida na aorta ascendente, também chamada de medida central da pressão arterial, parece ter diferentes valores e ser mais fidedigno do risco cardiovascular do que sua medida convencional, ou seja, a periférica. 
O componente elástico das artérias tem como função receber este fluxo ou jorradas de sangue, amortecê-las e propagá-las progressivamente. Com o envelhecimento, seja cronológico ou por adoecimento destas artérias, vai se perdendo as fibras elásticas e tendo como consequência endurecimento arterial (especialmente quando se trata da aórtica torácica). Este fenômeno é chamado de Arteriosclerose, o enrijecimento destes vasos com a idade é atribuível aos efeitos fatigantes do alongamento das paredes pelas pulsações com fratura da elastina, elemento fundamental para a acomodação do fluxo intermitente, vindo do ventrículo esquerdo para a aorta e as demais artérias. As fibras de elastina da aorta fraturam e a parede se estica, com tensões sendo progressivamente substituídas pelas fibras de colágeno. Estas fibras são mais resistentes, porém sem capacidade de acomodar o volume sistólico do ventrículo esquerdo. Isto é mais marcante na aorta ascendente. Estas alterações levam a duas modificações, a saber: I) aumento da VOP e/ou II) aumento do Alx. Efeitos nocivos do enrijecimento da aorta e seus ramos causam um maior aumento na pressão para um determinado pulso de fluxo, e é ampliado pela reflexão da onda, vindo da periferia que chega no retorno mais cedo, seja pelas bifurcações ou por outros fatores hemodinâmicos. Isto leva ao aumento da PAS na aorta e no ventrículo esquerdo e a PP, causando redução relativa na pressão de perfusão coronária.

Em consequência do envelhecimento e aumento da PAS e da PP central da aorta, ocorrem outros danos cardíacos e arteriais. Aumentos da PP em artérias de grande e médio calibre predispõem à progressão precoce de aterosclerose. $\mathrm{O}$ aumento das pulsações nas artérias centrais causa a mesma fadiga como visto na aorta, mas principalmente na artéria fisicamente fraca como nas bifurcações em geral e principalmente no cérebro, onde surgem pequenos aneurismas, com risco de ruptura e sangramento. As alterações hemodinâmicas descritas também favorecem o maior cisalhamento (desgaste por atrito) do endotélio, levando a disfunção deste componente da parede vascular. ${ }^{3}$

Assim, ocorre um círculo vicioso: fatores de risco cardiovascular aumentam a rigidez, as pressões arteriais e a hipertensão.

Em indivíduos jovens com paredes arteriais elásticas ainda preservadas, a PAS e a PP na aorta ascendente podem ser menores do que a PA medida no membro superior em até $20 \mathrm{mmHg}$. Em média, essa diferença é menor na população em geral e, em pacientes com hipertensão, gira em torno de 6-11 mmHg. A diferença entre a pressão central e a periférica se aplica ao componente pulsátil da PA, enquanto o componente estável (geralmente representado por PM) não muda significativamente ao longo da árvore arterial quando o indivíduo está na posição reclinada.

Foi demonstrado que a PAS central e a PP estão correlacionadas melhor com a extensão da aterosclerose coronária, espessura da íntima-média da carótida, hipertrofia ventricular esquerda, e função diastólica do ventrículo esquerdo, sendo todos estes marcadores de risco cardiovascular. Além disso, um número de estudos sugere correlação mais próxima de lesões nos órgão-alvo com PA central do que com PA periférica. Por um lado, placas ateroscleróticas difusas prejudicam as propriedades elásticas da parede arterial (embora placas instáveis, macias e ricas em lipídios não prejudicam a complacência arterial), enquanto, por outro lado, o aumento da rigidez aumenta o componente pulsátil da PA, levando à progressão das lesões ateroscleróticas. Isto leva a um círculo vicioso, que é difícil de prevenir ou quebrar na prática clínica. Vários estudos têm destacado o valor preditivo da PA central.

Além disso, o componente pulsátil da PA tem se mostrado um bom preditor de eventos cardiovasculares futuros, como a fração de ejeção ventricular esquerda, a extensão da aterosclerose coronária, diabetes e função renal. Uma recente meta-análise de cinco estudos demonstrou um maior valor preditivo da PAS central em comparação com PP periférico (com significância limítrofe), embora a PP central e periférica não tenham sido medidos simultaneamente sob as mesmas condições em todos os estudos incluídos na análise. ${ }^{3}$

Adicionalmente, os medicamentos para baixar a pressão arterial podem ter um efeito diferente sobre as pressões aórticas centrais e a hemodinâmica comparados através da PA braquial. De acordo com resultados do CAFÉ (Avaliação da Função da Artéria Conduíte), há evidências que a PA braquial não é um bom substituto para o efeito de drogas anti-hipertensivas na hemodinâmica arterial central. ${ }^{5}$

Ainda no referido estudo, apesar de um efeito semelhante na PA sistólica braquial entre os grupos de tratamento, aquele baseado em atenolol-tiazida foi muito menos eficaz do que o grupo que recebeu perindopril e amlodipina no que se refere a redução das pressões aórticas centrais. Uma das explicações destes efeitos diferentes relacionados aos diversos tratamentos nas pressões aórticas centrais pode ser o mecanismo potencial que também explica os divergentes resultados clínicos entre os dois braços de tratamento encontrados no ASCOT (o Anglo Escandinavo Teste de Resultados Cardíacos). ${ }^{6}$ Na contramão desses resultados, Mitchell et al. ${ }^{7}$ não encontraram uma significativa associação entre PP central e risco cardiovascular. Este fato pode ser causado pela diferença nas populações estudadas ou nos métodos utilizados para avaliar a PA central. Os últimos autores usaram tonometria de aplanação da artéria braquial, ao invés da habitual radial. Também é argumentado que o sinal é detectado através da aponeurose bicipital rígida enquanto a tonometria de aplanação realizada na artéria braquial. Metodologias bem diferentes.

As relações das pressões braquial e central com a hipertrofia da artéria carótida (espessura médio-intimal e massa vascular), extensão da aterosclerose (pontuação da placa) e eventos cardiovasculares incidentes foram examinadas no Strong Heart Study. ${ }^{8}$ As pressões centrais foram calculadas usando tonometria de aplanação radial. Entre 3.520 participantes, as PP central e braquial foram mais fortemente relacionadas à hipertrofia vascular e extensão da aterosclerose do que as pressões sistólicas. A pressão de pulso central foi mais fortemente relacionada a todas as três medidas arteriais do que a pressão de pulso braquial $(r=0,364$ versus 0,309 para pontuação de placa; $\mathrm{P}<0,001$ para comparação do coeficiente de correlação de Spearman; $r=0,293$ versus 0,249 para espessura médio-intimal; $\mathrm{P}<0,002 ; r=0,320$ versus 0,289 para massa vascular; $\mathrm{P}<0,05)$. Todos estes achados foram ajustados para os fatores de risco clássicos. Em conclusão, a pressão de pulso central determinada de forma não invasiva está mais fortemente relacionada à hipertrofia vascular, extensão da aterosclerose e eventos cardiovasculares 
do que a pressão arterial braquial. Esses achados apoiam o exame prospectivo do uso da pressão arterial central como alvo de tratamento em estudos futuros.

Achados recentes sugerem que o componente pulsátil da PA (quando representado pela pressão de pulso central ou pulsatilidade central) é um dos fatores mais importantes na determinação da sobrevida livre de eventos.

O estudo de coorte realizado por McEniery et al. pretendeu responder questões sobre o impacto relativo dos fatores de risco cardiovascular e doença ateromatosa na pressão central e a sua variação normal na pressão central em indivíduos saudáveis. ${ }^{9,10}$ As pressões sanguíneas sentadas periférica (braquial) e central (aórtica) foram avaliadas e a relação entre a pressão de pulso aórtica e braquial (razão de pressão de pulso, ou seja, 1 / amplificação) foi calculada em indivíduos saudáveis, diabéticos, portadores de doença cardiovascular e naqueles com apenas um dos seguintes: hipertensão, hipercolesterolemia ou tabagismo. Foram analisados dados de 10.613 indivíduos. Em comparação com indivíduos saudáveis, a PP aumentou significativamente (isto é, a pressão sistólica central foi relativamente mais alta) em indivíduos com fatores de risco ou doença ( $\mathrm{P}<0,01$ para todas as comparações). Embora o envelhecimento esteja associado a uma razão de pressão de pulso aumentada, ainda havia uma diferença média \pm DP entre a pressão sistólica braquial e aórtica de 11 \pm 4 e $8 \pm 3 \mathrm{mmHg}$ para homens e mulheres com idade> 80 anos, respectivamente. Esses dados demonstram que os fatores de risco cardiovascular afetam a relação da PP e que a pressão central não pode ser inferida de forma confiável a partir da pressão periférica. Assim, os resultados de vários estudos prospectivos (usando medidas invasivas e não invasivas da PA central) indicam não apenas um valor preditivo independente da pressão de pulso central, mas também sua vantagem sobre a pressão braquial. No entanto, grandes estudos prospectivos com o objetivo de comparar o valor preditivo da PA periférica e central na população em geral, bem como trabalhos comparando a eficácia dos diferentes tratamentos anti-hipertensivos, além de outros fatores de risco descritos com base nas medidas periféricas e centrais, são necessários antes de que algoritmos baseados na PA central possam ser recomendados para a prática clínica.

\section{CONCLUSÃO}

As medições das pressões centrais são mais fidedignas para estabelecimento de correlação com o risco cardiovascular do que as medidas periféricas. Contudo, seus equipamentos são mais caros e indisponíveis em muitos serviços de saúde neste momento.

\section{REFERÊNCIAS}

1. Palatini P, Casiglia E, Gąsowski J, et al. Arterial stiffness, central hemodynamics, and cardiovascular risk in hypertension. Vasc Health Risk Manag. 2011; 7:725-39. doi: 10.2147/VHRM.S25270. Epub 2011 Dec 7.

2. Wang KL, Cheng HM, Chuang SY, et al. Central or peripheral systolic or pulse pressure: which best relates to target organs and future mortality? J Hypertens. 2009; 27(3):461-467.

3. Mitchell GF. Early vascular modifications in hypertension: pathophysiological considerations.Medicographia. 2015;37( 4):379-82.

4. Brandão A. Amodeo C. Alcantara C, et al. Posicionamento Luso-Brasileiro de Pressão Arterial Central. Arq Bras Cardiol. 2017;108(2):100-8.

5. Williams B. Saver SL Thom SM, et al. The CAFE Investigators and Steering Committee and Writing for the Anglo-Scandinavian Cardiac Outcomes Trial (ASCOT) Investigators. Differential impact of blood pressure-lowering drugs on central aortic pressure and clinical outcomes: principal results of the Conduit Artery Function Evaluation (CAFE) study. Circulation. 2006:7;113(9):1213-25. doi: 10.1161/CIRCULATIONAHA.105.595496.

6. Rothwell PM, Howard SC, Dolan E, et al.; ASCOT-BPLA and MRC Trial

Investigators. Effects of beta blockers and calcium-channel blockers on within-individual variability in blood pressure and risk of stroke. Lancet Neurol. 2010;9:469-80. doi: 10.1016/S1474-4422(10)70066-1.

7. Mitchell GF, Hwang SJ, Vasan RS, et al. Arterial stiffness and cardiovascular events: the Framingham Heart Study. Circulation. 2010;121(4):505-11. doi: 10.1161/CIRCULATIONAHA.109.886655.

8. Roman MJ. Kizer JR. Galloway JM, et al Central Pressure More Strongly Relates to Vascular Disease and Outcome Than Does Brachial Pressure The Strong Heart Study. Hypertension.2007;50:197-203. doi: 10.1161/ HYPERTENSIONAHA.107.089078.

9. Safar ME, Jankowski P. Central blood pressure and Hypertension: role in cardiovascular risk assessment. Clin Sci (Lond).2009;116(4):273-82. doi: $10.1042 / \operatorname{cs} 20080072$

10. McEniery CM, Yasmin, Wallace $S$, et al. Increased stroke volume and aortic stiffness contribute to isolated systolic hypertension in young adults. Hypertension.2005;46:221-6. doi: 10.1161/01. HYP.0000165310.84801.e0. 